\title{
Prime Cordial Labeling of Some Graphs
}

\author{
Samir K. Vaidya ${ }^{1}$, Nirav H. Shah ${ }^{2}$ \\ ${ }^{1}$ Saurashtra University, Rajkot, India \\ ${ }^{2}$ V.V.P. Engineering College, Rajkot, India \\ Email: samirkvaidya@yahoo.co.in,nirav.hs@gmail.com
}

Received October 1, 2011; revised November 9, 2011; accepted November 16, 2011

\begin{abstract}
In this paper we prove that the split graphs of $K_{1, n}$ and $B_{n, n}$ are prime cordial graphs. We also show that the square graph of $B_{n, n}$ is a prime cordial graph while middle graph of $P_{n}$ is a prime cordial graph for $n \geq 4$. Further we prove that the wheel graph $W_{n}$ admits prime cordial labeling for $n \geq 8$.
\end{abstract}

Keywords: Prime Cordial Labeling; Split Graph; Square Graph; Middle Graph

\section{Introduction}

We begin with simple, finite, connected and undirected graph $G=(V(G), E(G))$ with $p$ vertices and $q$ edges. For standard terminology and notations we follow Gross and Yellen [1]. We will provide brief summary of definitions and other information which are necessary for the present investigations.

Definition 1.1 If the vertices are assigned values subject to certain condition(s) then it is known as graph labeling.

Any graph labeling will have the following three common characteristics:

1) A set of numbers from which vertex labels are chosen;
2) A rule that assigns a value to each edge;

3) A condition that this value has to satisfy.

According to Beineke and Hegde [2] graph labeling serves as a frontier between number theory and structure of graphs. For a dynamic survey of various graph labeling problems along with extensive bibliography we refer to Gallian [3].

Definition 1.2 A mapping $f: V(G) \rightarrow\{0,1\}$ is called binary vertex labeling of $G$ and $f(v)$ is called the label of the vertex $v$ of $G$ under $f$.

Notation 1.3 If for an edge $e=u v$, the induced edge labeling $f^{*}: E(G) \rightarrow\{0,1\}$ is given by

$$
f^{*}(e)=|f(u)-f(v)| \text {. Then }
$$

$$
\left.\begin{array}{l}
v_{f}(i)=\text { number of vertices of } G \text { having label } i \text { under } f \\
e_{f}(i)=\text { number of edges of } G \text { having label } i \text { under } f^{*}
\end{array}\right\} \text { where } i=0 \text { and } 1
$$

Definition 1.4 A binary vertex labeling $f$ of a graph $G$ is called a cordial labeling if $\left|v_{f}(0)-v_{f}(1)\right| \leq 1$ and $\left|e_{f}(0)-e_{f}(1)\right| \leq 1$. A graph $G$ is cordial if it admits cordial labeling.

The concept of cordial labeling was introduced by Cahit [4]. Some labeling schemes are also introduced with minor variations in cordial theme. Some of them are product cordial labeling, total product cordial labeling and prime cordial labeling. The present work is focused on prime cordial labeling.

Definition 1.5 A prime cordial labeling of a graph $G$ with vertex set $V(G)$ is a bijection

$f: V(G) \rightarrow\{1,2,3, \cdots, \mid V(G)\}$ and the induced function $f^{*}: E(G) \rightarrow\{0,1\}$ is defined by

$$
f *(e=u v)=\left\{\begin{array}{l}
1 ; \text { if } \operatorname{gcd}(f(u), f(v))=1 \\
0 ; \text { otherwise }
\end{array}\right.
$$

satisfies the condition $\mid e_{f}(0)-e_{f}(1) \leq 1$. A graph which admits prime cordial labeling is called a prime cordial graph.

The concept of prime cordial labeling was introduced by Sundaram [5] et al. and in the same paper they have investigated several results on prime cordial labeling. Vaidya and Vihol [6] have also discussed prime cordial labeling in the context of graph operations while in [7] the same authors have discussed prime cordial labeling for some cycle related graphs. Vaidya and Shah [8] have investigated many results on this concept. In the present paper we obtain some new prime cordial graphs. 
Definition 1.6 Bistar is the graph obtained by joining the apex vertices of two copies of star $K_{1, n}$.

Definition 1.7 For a graph $G$ the split graph is obtained by adding to each vertex $v$ a new vertex $v^{\prime}$ such that $v^{\prime}$ is adjacent to every vertex that is adjacent to $v$ in $G$. The resultant graph is denoted as $\operatorname{spl}(G)$.

Definition 1.8 For a simple connected graph $G$ the square of graph $G$ is denoted by $G^{2}$ and defined as the graph with the same vertex set as of $G$ and two vertices are adjacent in $G^{2}$ if they are at a distance 1 or 2 apart in $G$.

Definition 1.9 The middle graph $M(G)$ of a graph $G$ is the graph whose vertex set is $V(G) \cup E(G)$ and in which two vertices are adjacent if and only if either they are adjacent edges of $G$ or one is a vertex of $G$ and the other is an edge incident with it.

\section{Main Results}

Theorem $2.1 \operatorname{spl}\left(K_{1, n}\right)$ is a prime cordial graph.

Proof: Let $v_{1}, v_{2}, v_{3}, \cdots, v_{n}$ be the pendant vertices, $v$ be the apex vertex of $K_{1, n}$ and $u, u_{1}, u_{2}, u_{3}, \cdots, u_{n}$ are the vertices corresponding to $v, v_{1}, v_{2}, v_{3}, \cdots, v_{n}$ in $\operatorname{spl}\left(K_{1, n}\right)$. Denoting $\operatorname{spl}\left(K_{1, n}\right)=G$ then $|V(G)|=2 n+2$ and $|E(G)|=3 n$.

To define $f: V(G) \rightarrow\{1,2,3, \cdots, 2 n+2\}$, we consider following two cases.

Case 1: $n=2,3$

The graphs $\operatorname{spl}\left(K_{1,2}\right)$ and $\operatorname{spl}\left(K_{1,3}\right)$ are to be dealt separately and their prime cordial labeling is shown in Figure 1.

Case 2: $n \geq 4$

$$
\begin{array}{ll}
f(v)=4, & \\
f(u)=2, & \\
f\left(v_{i}\right)=2 i+4 ; & 1 \leq i \leq\left\lfloor\frac{n}{2}\right\rfloor+1 \\
f\left(v_{\left\lfloor\frac{n}{2}\right\rfloor+1+i}\right)=2 i-1 ; & 1 \leq i \leq\left\lceil\frac{n-2}{2}\right\rceil \\
f\left(u_{i}\right)=f\left(v_{n}\right)+2 i ; & 1 \leq i \leq n+1-\left\lceil\frac{n-2}{2}\right\rceil \\
f\left(u_{n+1-i}\right)=2(n+2-i) ; & 1 \leq i \leq\left\lceil\frac{n-4}{2}\right\rceil
\end{array}
$$

In view of the labeling pattern defined above we have $e_{f}(0)=\frac{3 n}{2}=e_{f}(1)$ (for $n$ even) and $e_{f}(0)=\left\lfloor\frac{3 n}{2}\right\rfloor=e_{f}(1)-1$ (for $n$ odd). Thus we have $\left|e_{f}(0)-e_{f}(1)\right| \leq 1$.

Hence $G$ is a prime cordial graph.

Illustration 2.2 Prime cordial labeling for $\operatorname{spl}\left(K_{1,7}\right)$ is shown in Figure 2.
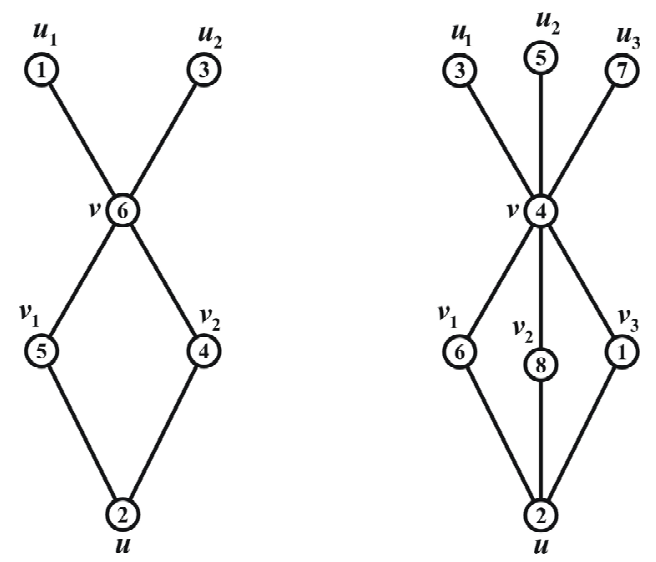

Figure 1. $\operatorname{spl}\left(K_{1,2}\right), \operatorname{spl}\left(K_{1,3}\right)$ and their prime cordial labelling.

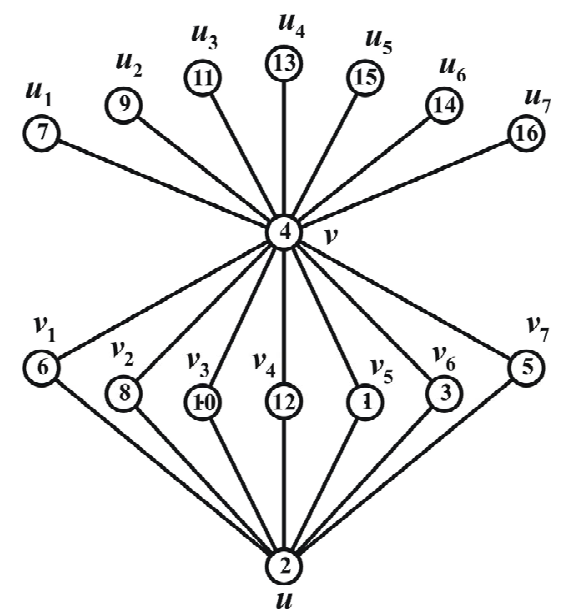

Figure 2. $\operatorname{spl}\left(K_{1,7}\right)$ and its prime cordial labelling.

Theorem $2.3 \operatorname{spl}\left(B_{n, n}\right)$ is a prime cordial graph.

Proof: Consider $B_{n, n}$ with vertex set

$\left\{u, v, u_{i}, v_{i}, 1 \leq i \leq n\right\}$ where $u_{i}, v_{i}$ are pendant vertices. In order to obtain $\operatorname{spl}\left(B_{n, n}\right)$ add $u^{\prime}, v^{\prime}, u_{i}^{\prime}, v_{i}^{\prime}$ vertices corresponding to $u, v, u_{i}, v_{i}$ where $1 \leq i \leq n$. If $G=\operatorname{spl}\left(B_{n, n}\right)$ then $|V(G)|=4(n+1)$ and $|E(G)|=6 n+3$. We define vertex labeling $f: V(G) \rightarrow\{1,2,3, \cdots, 4(n+1)\}$ as follows.

$$
\begin{array}{ll}
f(u)=6, & \\
f(v)=2, & \\
f\left(u^{\prime}\right)=4, & \\
f\left(v^{\prime}\right)=1, & \\
f\left(u_{i}\right)=8+2(i-1) ; & 1 \leq i \leq n \\
f\left(u_{i}^{\prime}\right)=f\left(u_{n}\right)+2 i ; & 1 \leq i \leq n-1 \\
f\left(u_{n}^{\prime}\right)=3, & \\
f\left(v_{i}\right)=5+2(i-1) ; & 1 \leq i \leq n \\
f\left(v_{i}^{\prime}\right)=f\left(v_{n}\right)+2 i ; & 1 \leq i \leq n
\end{array}
$$


In view of pattern defined above we have

$e_{f}(0)=3 n+2=e_{f}(1)+1$.

That is, $\left|e_{f}(0)-e_{f}(1)\right| \leq 1$.

Hence $G$ is a prime cordial graph.

Illustration 2.4 Prime cordial labeling of the graph $\operatorname{spl}\left(B_{5,5}\right)$ is shown in Figure 3.

Theorem $2.5 B_{n, n}^{2}$ is a prime cordial graph.

Proof: Consider $B_{n, n}$ with vertex set $\left\{u, v, u_{i}, v_{i}, 1 \leq i \leq n\right\}$ where $u_{i}, v_{i}$ are pendant vertices. Let $G$ be the graph $B_{n, n}^{2}$ then $|V(G)|=2 n+2$ and $|E(G)|=4 n+1$.

To define $f: V(G) \rightarrow\{1,2,3, \cdots, 2 n+2\}$, we consider following two cases.

Case 1: $n=2,3$

The graphs $B_{2,2}^{2}$ and $B_{3,3}^{2}$ are to be dealt separately and their prime cordial labeling is shown in Figure 4.
Case 2: $n \geq 4$

Choose a prime number $\mathrm{p}$ such that $3 p \leq 2 n+2<5 p$,

$$
\begin{aligned}
& f(u)=2, \\
& f\left(u_{1}\right)=1,
\end{aligned}
$$

$f\left(u_{i}\right)=m$, where $m$ is distinct even numbers between 4 and $2 n+2$ except $2 p$ with $2 \leq i \leq n$.

$$
f(v)=2 p,
$$

$f\left(v_{i}\right)=3+2(i-1)$ for $1 \leq i \leq n$.

In view of the above defined labeling pattern we have $e_{f}(0)-1=2 n=e_{f}(1)$.

Thus in both the cases we have $\left|e_{f}(0)-e_{f}(1)\right| \leq 1$.

Hence $G$ is a prime cordial graph.

Illustration 2.6 Prime cordial labeling of the graph $B_{7,7}^{2}$ is shown in Figure 5.

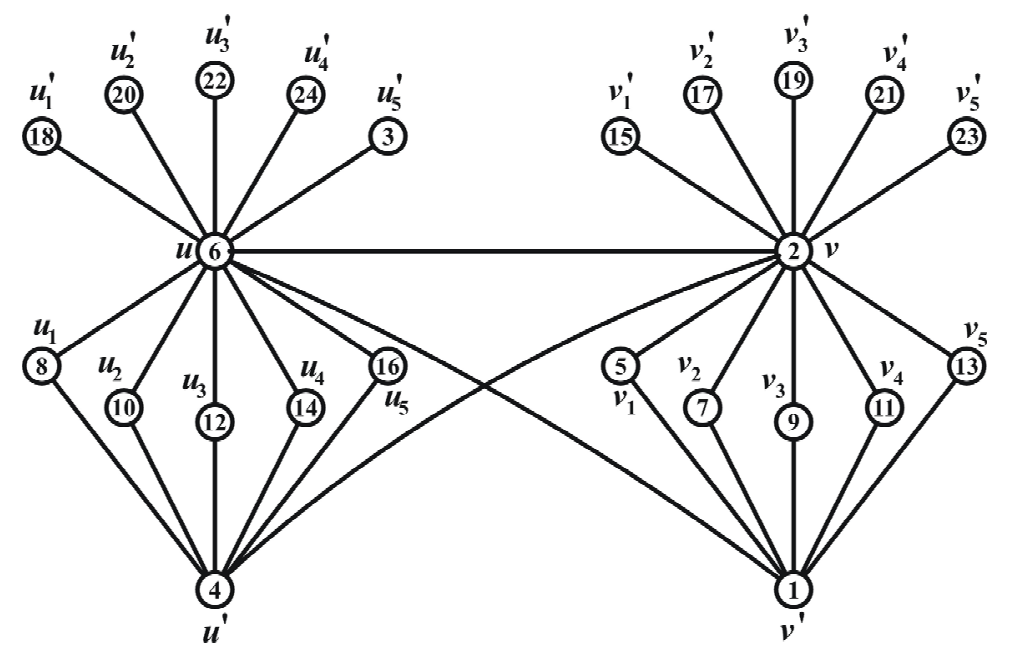

Figure 3. $\operatorname{spl}\left(B_{5,5}\right)$ and its prime cordial labelling.
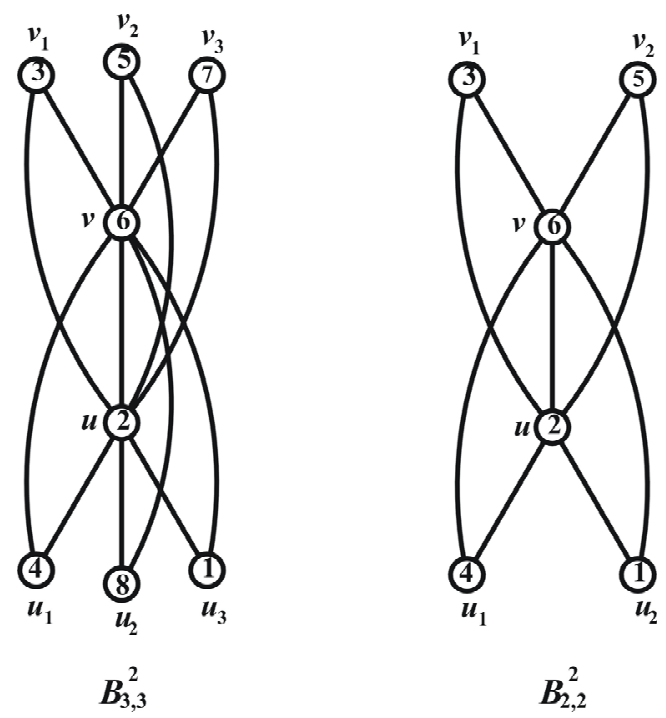

Figure 4. $B_{2,2}^{2}, \quad B_{3,3}^{2}$ and their prime cordial labelling. 
Theorem 2.7 $M\left(P_{n}\right)$ is not a prime cordial graph for $n=2,3$.

Proof: Let $v_{1}, v_{2}, \cdots, v_{n}$ and $e_{1}, e_{2}, \cdots, e_{n-1}$ are respectively be the vertices and edges of $P_{n}$. Add vertices $v_{1}^{\prime}, v_{2}^{\prime}, \cdots, v_{n-1}^{\prime}$ corresponding to the edges $e_{1}, e_{2}, \cdots, e_{n-1}$ in order to obtain middle graph of $P_{n}$. Let $G$ be the graph $M\left(P_{n}\right)$. Then $|V(G)|=2 n-1$ and $|E(G)|=3 n-4$.

For the graph $M\left(P_{2}\right)$ the possible assignment of labels to adjacent vertices are $(1,2),(1,3),(2,3)$. Such assignment will generate no edge with label 0 and two edges with label 1. That is, $\left|e_{f}(0)-e_{f}(1)\right|=2>1$. Therefore $M\left(P_{2}\right)$ is not a prime cordial graph.

For the graph $M\left(P_{3}\right)$ the possible assignment of labels to adjacent vertices are $(1,2),(1,3),(1,4),(1,5),(2,3)$, $(2,4),(2,5),(3,4),(3,5)$. Such assignment will generate maximum one edge with label 0 and minimum four edges with label 1 . That is, $\left|e_{f}(0)-e_{f}(1)\right|=3>1$. Therefore $M\left(P_{3}\right)$ is not a prime cordial graph.

Hence $G$ is not a prime cordial graph for $n=2,3$.

Theorem 2.8 $M\left(P_{n}\right)$ is a prime cordial graph for $n \geq 4$.

Proof: Let $v_{1}, v_{2}, \cdots, v_{n}$ and $e_{1}, e_{2}, \cdots, e_{n-1}$ are respectively be the vertices and edges of $P_{n}$. Add vertices $v_{1}^{\prime}, v_{2}^{\prime}, \cdots, v_{n-1}^{\prime}$ corresponding to the edges $e_{1}, e_{2}, \cdots, e_{n-1}$ in order to obtain middle graph of $P_{n}$. Let $G$ be the graph $M\left(P_{n}\right)$. Then $|V(G)|=2 n-1$ and $|E(G)|=3 n-4$. To define

$f: V(G) \rightarrow\{1,2,3, \cdots, 2 n-1\}$, we consider following two cases.

Case 1: $n$ is even, $n \geq 4$.

$$
\begin{aligned}
& f\left(v_{1}\right)=1, \\
& f\left(v_{2}\right)=4, \\
& f\left(v_{2+i}\right)=10+4(i-1) ; \quad 1 \leq i \leq \frac{n}{2}-2 \\
& f\left(\begin{array}{c}
v_{\frac{n}{2}+i} \\
2
\end{array}\right)=3+4(i-1) ; \quad 1 \leq i \leq \frac{n}{2} \\
& f\left(v_{1}^{\prime}\right)=2, \\
& f\left(v_{1+i}^{\prime}\right)=8+4(i-1) ; \quad 1 \leq i \leq \frac{n}{2}-2 \\
& f\left(\begin{array}{c}
v_{\frac{n}{2}}^{\prime} \\
2
\end{array}\right)=6, \\
& f\left(\begin{array}{c}
v_{\frac{n}{2}+i}^{\prime} \\
\frac{1}{2}
\end{array}\right)=5+4(i-1) ; \quad 1 \leq i \leq \frac{n}{2}-1
\end{aligned}
$$

In view of the above defined labeling pattern we have $e_{f}(0)=\frac{3 n}{2}-2=e_{f}(1)$.

Case 2: $n$ is odd, $n \geq 5$

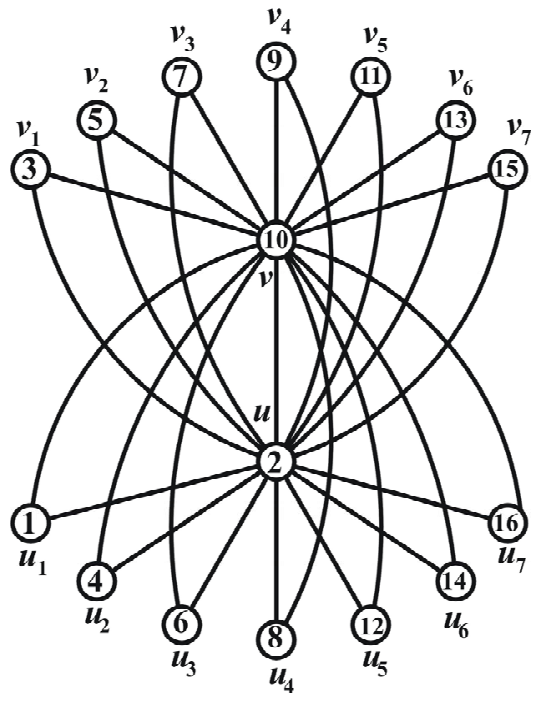

Figure 5. $B_{7,7}^{2}$ and its prime cordial labelling.

$$
\begin{gathered}
f\left(v_{1}\right)=1, \\
f\left(v_{2}\right)=4, \\
f\left(v_{2+i}\right)=10+4(i-1) ; \quad 1 \leq i \leq\left\lfloor\frac{n}{2}\right\rfloor-2 \\
f\left(v_{\left.\left\lceil\frac{n}{2}\right\rceil\right)=6,}\right. \\
f\left(v_{\left\lceil\frac{n}{2}\right\rceil+i}\right)=5+4(i-1) ; \quad 1 \leq i \leq\left\lfloor\frac{n}{2}\right\rfloor \\
f\left(v_{i+1}^{\prime}\right)=8+4(i-1) ; \quad 1 \leq i \leq\left\lfloor\frac{n}{2}\right\rfloor-1 \\
f\left(v_{1}^{\prime}\right)=2, \quad \\
f\left(v_{\left\lfloor\frac{n}{2}\right\rfloor+i}^{\prime}\right)=3+4(i-1) ; \quad 1 \leq i \leq\left\lfloor\frac{n}{2}\right\rfloor
\end{gathered}
$$

In view of the above defined labeling pattern we have $e_{f}(0)=\left\lfloor\frac{3 n-4}{2}\right\rfloor=e_{f}(1)-1$.

Thus in both the cases we have $\left|e_{f}(0)-e_{f}(1)\right| \leq 1$.

Hence $G$ is a prime cordial graph for $n \geq 4$.

Illustration 2.9 Prime cordial labeling of the graph $M\left(P_{11}\right)$ is shown in Figure 6.

Theorem 2.10 $W_{n}$ is not a prime cordial graph for $n=$ 3 to $n=7$.

Proof: Let $v_{0}$ be the apex vertex of wheel $W_{n}$ and $v_{1}, v_{2}, \cdots, v_{n}$ be the rim vertices. Then $\left|V\left(W_{n}\right)\right|=n+1$ and $\left|E\left(W_{n}\right)\right|=2 n$.

For the graph $W_{3}$ the possible pairs of labels of adja- 


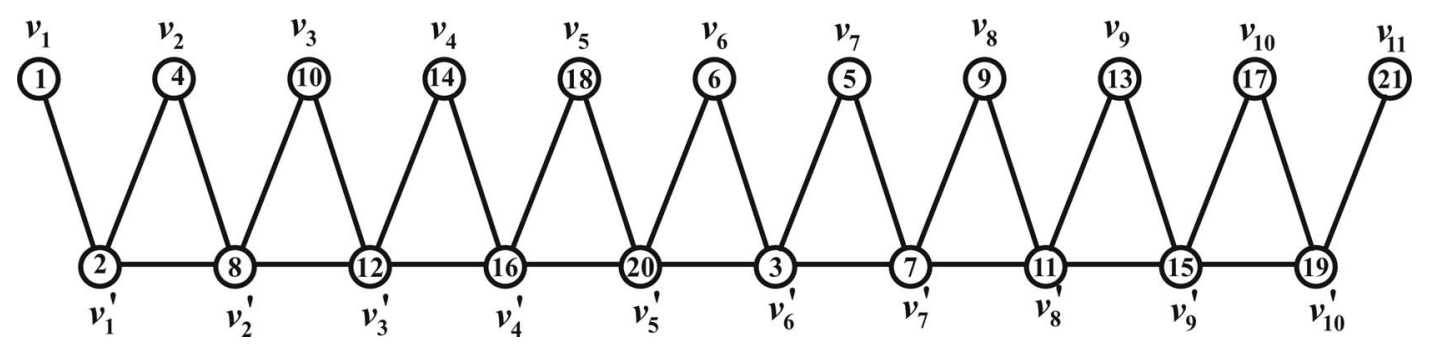

Figure 6. $M\left(P_{11}\right)$ and its prime cordial labelling.

cent vertices will be $(1,2),(1,3),(1,4),(2,3),(2,4),(3,4)$. Such assignment will generate maximum one edge with label 0 and minimum five edges with label 1 . That is, $\left|e_{f}(0)-e_{f}(1)\right|=4>1$. Hence $W_{3}$ is not a prime cordial graph.

For the graph $W_{4}$ the possible assignment of labels to adjacent vertices will be $(1,2),(1,3),(1,4),(1,5),(2,3)$, $(2,4),(2,5),(3,4),(3,5),(4,5)$. Such assignment will generate maximum one edge with label 0 and minimum seven edges with label 1 . That is, $\left|e_{f}(0)-e_{f}(1)\right|=6>1$. Hence $W_{4}$ is not a prime cordial graph.

For the graph $W_{5}$ the possible assignment of labels to adjacent vertices will be $(1,2),(1,3),(1,4),(1,5),(1,6)$, $(2,3),(2,4),(2,5),(2,6),(3,4),(3,5),(3,6),(4,5),(4,6)$, $(5,6)$. Such assignment will generate maximum four edges with label 0 and minimum six edges with label 1 . That is, $\left|e_{f}(0)-e_{f}(1)\right|=2>1$. Hence $W_{5}$ is not a prime cordial graph.

For the graph $W_{6}$ the possible assignment of labels to adjacent vertices will be $(1,2),(1,3),(1,4),(1,5),(1,6)$, $(1,7),(2,3),(2,4),(2,5),(2,6),(2,7),(3,4),(3,5),(3,6)$, $(3,7),(4,5),(4,6),(4,7),(5,6),(5,7),(6,7)$. Such assignment will generate maximum four edges with label 0 and minimum eight edges with label 1 . That is,

$\left|e_{f}(0)-e_{f}(1)\right|=4>1$. Hence $W_{6}$ is not a prime cordial graph.

Now in $W_{7}$ to satisfy the edge condition for prime cordial labeling it is essential to label seven edges with label 0 and seven edges with label 1 out of fourteen edges. But all the possible assignments of vertex labels will give rise to 0 labels for at most six edges and 1 labels for at least eight edges. That is, $\left|e_{f}(0)-e_{f}(1)\right|=2>1$. Hence $W_{7}$ is not a prime cordial graph.

Hence $W_{n}$ is not a prime cordial graph for $n=3$ to $n=7$.

Theorem 2.11 $W_{n}$ is a prime cordial graph for $n \geq 8$.

Proof: Let $v_{0}$ be the apex vertex of wheel $W_{n}$ and $v_{1}, v_{2}, \cdots, v_{n}$ be the rim vertices. To define

$f: V\left(W_{n}\right) \rightarrow\{1,2,3, \cdots, 2 n\}$, we consider the following three cases.

Case 1: $n=8,9,10$

The graphs $W_{8}, W_{9}$ and $W_{10}$ are to be dealt separately and their prime cordial labeling is shown Figure 7.

Case 2: $n$ is even, $n \geq 12$

$$
\begin{aligned}
& f\left(v_{0}\right)=2 \text {, } \\
& f\left(v_{1}\right)=5 \text {, } \\
& f\left(v_{2}\right)=10 \text {, } \\
& f\left(v_{3}\right)=4 \text {, } \\
& f\left(v_{4}\right)=8 \text {, } \\
& f\left(v_{4+i}\right)=12+2(i-1) ; \quad 1 \leq i \leq \frac{n}{2}-5 \\
& f\left(v_{\frac{n}{2}}\right)=6 \\
& f\left(v_{\frac{n}{2}+1}\right)=3 \text {, } \\
& f\left(v_{\frac{n}{2}+2}\right)=9 \text {, } \\
& f\left(v_{n-1}\right)=1 \text {, } \\
& f\left(v_{n}\right)=7 \text {, } \\
& f\left(v_{\frac{n}{2}+2+i}\right)=11+2(i-1) ; \quad 1 \leq i \leq \frac{n}{2}-4
\end{aligned}
$$

In view of the above defined labeling pattern we have $e_{f}(0)=n=e_{f}(1)$.

Case 3: $n$ is odd, $n \geq 11$

$$
\begin{aligned}
& f\left(v_{0}\right)=2, \\
& f\left(v_{1}\right)=10, \\
& f\left(v_{2}\right)=4, \\
& f\left(v_{3}\right)=8, \\
& f\left(v_{3+i}\right)=12+2(i-1) ; 1 \leq i \leq \frac{n-1}{2}-4 \\
& f\left(v_{\frac{n-1}{2}}\right)=6, \\
& f\left(v_{\frac{n+1}{2}}\right)=3, \\
& f\left(v_{\frac{n+3}{2}}\right)=1, \\
& f\left(v_{n+1-i}\right)=5+2(i-1) ; 1 \leq i \leq \frac{n-3}{2}
\end{aligned}
$$




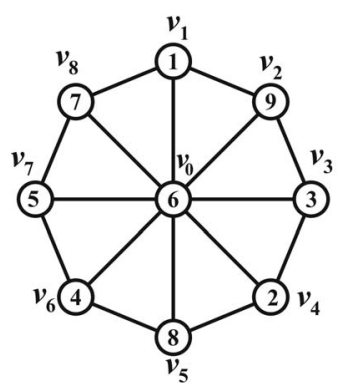

$\mathrm{W}_{8}$

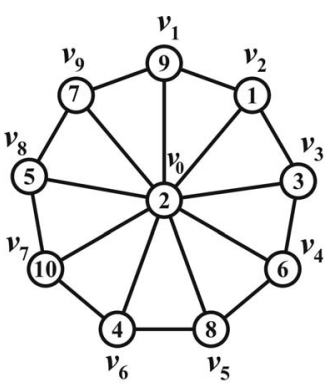

$\mathbf{W}_{9}$

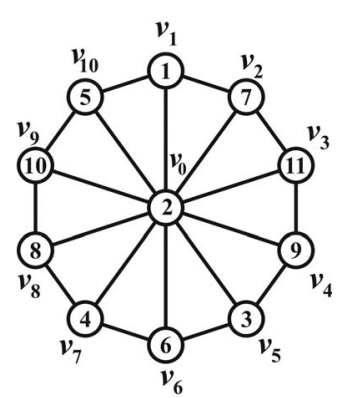

$\mathrm{W}_{10}$

Figure 7. $W_{8}, W_{9}$ and $W_{10}$ and their prime cordial labelling.

In view of the above defined labeling pattern we have $e_{f}(0)=n=e_{f}(1)$.

Thus in all the cases we have $\left|e_{f}(0)-e_{f}(1)\right| \leq 1$.

Hence $W_{n}$ is a prime cordial graph for $n \geq 8$.

Illustration 2.12 Prime cordial labeling of the graph $W_{13}$ is shown in Figure 8.

\section{Concluding Remarks}

As not every graph admit prime cordial labeling it is very interesting to investigate graph or graph families which admit prime cordial labeling. In this paper we have investigated some new prime cordial graphs. To investigate similar results for other graph families as well as in the context of different labeling problems is an open area of research.

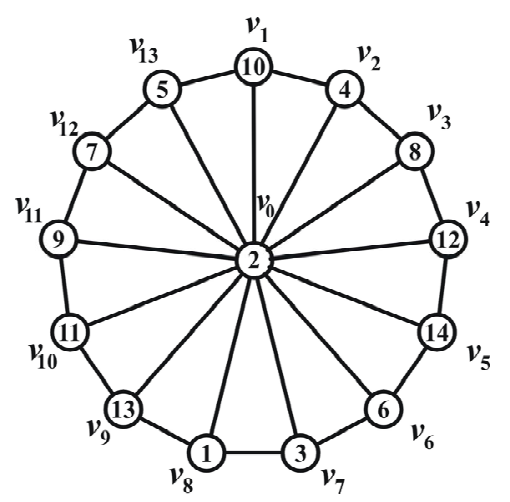

Figure 8. $W_{13}$ and its prime cordial labelling.

\section{REFERENCES}

[1] J. Gross and J. Yellen, "Graph Theory and Its Applications,” CRC Press, Boca Raton, 1999.

[2] L. W. Beineke and S. M. Hegde, "Strongly Multiplicative Graphs,” Discussiones Mathematicae Graph Theory, Vol. 21, 2001, pp. 63-75.

[3] J. A. Gallian, “A Dynamic Survey of Graph Labeling,” The Electronic Journal of Combinatorics, Vol. 17, 2010, DS6. http://www.combinatorics.org/Surveys/ds6.pdf

[4] I. Cahit, "Cordial Graphs: A Weaker Version of Graceful and Harmonious Graphs,” Ars Combinatoria, Vol. 23, 1987, pp. 201-207.

[5] M. Sundaram, R. Ponraj and S. Somasundram, "Prime Cordial Labeling of Graphs," Journal of the Indian Academy of Mathematics, Vol. 27, No. 2, 2005 , pp. 373390.

[6] S. K. Vaidya and P. L. Vihol, "Prime Cordial Labeling for Some Graphs,” Modern Applied Science, Vol. 4, No. 8, 2010, pp. 119-126.

[7] S. K. Vaidya and P. L. Vihol, "Prime Cordial Labeling for Some Cycle Related Graphs,” International Journal of Open Problems in Computer Science and Mathematics, Vol. 3, No. 5, 2010, pp. 223-232.

[8] S. K. Vaidya and N. H. Shah, "Some New Families of Prime Cordial Graphs,” Journal of Mathematics Research, Vol. 3, No. 4, 2011, pp. 21-30. doi:10.5539/jmr.v3n4p21 